\title{
Razones del Incumplimiento de los Controles de Seguridad en el Trabajo en Empresas Colombianas
}

\section{REASONS OF THE BREACH OF THE SAFETY CONTROLS IN THE WORK IN COLOMBIAN COMPANIES}

\author{
Ninfa del Carmen Vega-Monsalve
}

Docente-Investigadora de la Corporación Universitaria Minuto de Dios-UNIMINUTO. Bello, Colombia.

\section{RESUMEN}

Objetivo: Conocer las razones del incumplimiento de los controles de seguridad en el trabajo en empresas colombianas.

Método: Estudio transversal con una muestra de 675 gestores de seguridad y salud en el trabajo de 120 municipios del país. Se realizaron igual número de encuestas, tres grupos focales y nueve entrevistas. Para los datos cuantitativos se efectuaron cálculos estadísticos descriptivos; para los cualitativos análisis del discurso, identificación y clasificación estructural de dimensiones estratégicas y búsqueda de patrón de comportamiento común.

Resultados: Se encontró que el 23,11\% de los profesionales consultados, durante el último año, casi siempre estuvo frustrado por no poder implementar los controles de seguridad laboral que sabía requería la empresa donde labora. Entre otras, las principales razones fueron: con un 31\% falta de apoyo de la gerencia y un $27,1 \%$ falta de apoyo del resto de coordinadores.

Conclusiones: El sistema de gestión en seguridad y salud en el trabajo requiere una visión más estratégica por parte de las empresas y una acción más convincente por parte de los profesionales del área.

(Vega-Monsalve N, 2016. Razones del Incumplimiento de los Controles de Seguridad en el Trabajo en Empresas Colombianas. Cienc Trab. SepDic; 18 [57]: 154-158).

Palabras claves: SALUD LABORAL, SEGURIDAD EN EL TRABAJO, ORGANIZACIÓN INDUSTRIAL, COMPORTAMIENTO DE LAS EMPRESAS, SISTEMAS ADMINISTRATIVOS, GESTIÓN.

\section{ABSTRACT}

Objective: To describe the reasons of the breach of the safety controls in the work in Colombian companies.

Method: Cross-sectional study with a sample of 675 managers of safety and health in the work of 120 municipalities of Colombian. They were realised equal number of focal surveys, three groups and nine interviews. Indicator of 3,74\% of error and 95\% of confidence. Descriptive statistical calculations took place.

Results: One was that the $23,11 \%$ of the professionals consulted, during the last year were frustrated almost always, by not being able to implement the job safety controls that knew required the company where it toils. Among others, the main reasons were: With a $31 \%$ lack of support of the management and 27,1\% lack of support of the rest of coordinators.

Conclusions: The system of management in safety and health in the work requires a more strategic vision on the part of the companies and a more convincing action on the part of the professionals of the area.

Keywords: OCCUPATIONAL HEALTH; INDUSTRIAL ORGANISATION; FIRM BEHAVIOR; ORGANIZATION AND ADMINISTRATION; MANAGEMENT.

\section{INTRODUCCIÓN}

Los controles de seguridad laboral son las decisiones y acciones adoptadas por la empresa para proteger la vida de su personal frente al oficio que desempeñan. ${ }^{1,2,3}$ Hablar de seguridad industrial es igual a productividad del negocio ${ }^{4}$ y bienestar del personal, de allí la importancia de que los controles sean implementados de forma efectiva.

Correspondencia / Correspondence:

Ninfa del Carmen Vega-Monsalve

Km. 0 Autopista Medellin - Bogotá. Bello, Colombia

Tel: 057-3148941227

e-mail: ninfavegamonsalve@gmail.com

Recibido: 17 de Julio de 2016 / Aceptado: 21 de Noviembre de 2016
Se estima que cada año a nivel mundial los riesgos laborales provocan la muerte de más de 2,3 millones de trabajadores. ${ }^{5}$ En Colombia, a pesar del endurecimiento de las sanciones, actualización de la normatividad y de que el 52,7\% de las empresas ejecutan actividades de bajo riesgo, ${ }^{6}$ se presenta una tasa de Accidentes Laborales (AL) de 7,73 por cada 100 afiliados, ${ }^{7}$ siendo en 2014 más alta que en años anteriores.

Diversos estudios han evidenciado que estos índices están relacionados con la escasa implementación del Sistema de Gestión en Seguridad y Salud Laboral (SG-SST) o su visión reduccionista y poco estratégica. ${ }^{8,9}$ Este hecho es ratificado por los órganos de control, según los cuales aún falta en materia de implementación por parte de las empresas. Por ejemplo, ${ }^{10}$ solo el 21,07\% de las empresas realiza programas de vigilancia epidemiológica y el $55,5 \%$ tiene panorama de factores de riesgo.

Además de que existe un principio de responsabilidad social empresarial de cuidar la vida de los trabajadores ${ }^{11}$, incluir el SG-SST en la planeación estratégica asegura una ventaja compe- 
titiva sostenible y resultados más eficientes en todos los procesos. $^{12,13}$ Para ello se requiere el concurso de todos los niveles de la empresa y el liderazgo decidido del Gestor de Seguridad y Salud en el Trabajo (G-SST), quien debe estar motivado y respaldado en su gestión. ${ }^{14}$

A pesar de que la literatura internacional refiere ciertas prácticas que favorecen el SG-SST, como por ejemplo: contar con el papel decidido de los directivos y su liderazgo transformacional ${ }^{15}$, que el G-SST sea competente para el rol $^{14}$, que haya presión de los grupos de interés, regulaciones estatales y cultura organizacional ${ }^{16}$ y que los trabajadores tengan comportamientos seguros. ${ }^{17}$

Son escasos los estudios que señalen las causas de la baja implementación del sistema, al menos en el escenario colombiano. Se encontró solo uno que, al indagar a 75 empresarios del departamento de Antioquia, encontró que la baja implementación de los controles de seguridad se daba por falta de presupuesto y compromiso de los equipos de trabajo.

De allí la relevancia y novedad de esta investigación que pretende responder la pregunta: ¿cuáles son las razones del incumplimiento de los controles de seguridad en el trabajo en empresas colombianas?; es conveniente informar que este estudio hace parte de un macroproyecto llamado: Competencias laborales y gestión de riesgos laborales.

\section{MÉTODO}

Este estudio aprovechó las bondades de los dos tipos de investigación (cualitativa y cuantitativa). Al no disponer de información sobre las posibles razones del incumplimiento de los controles de seguridad en el trabajo en las empresas del país, se decidió realizar desde el enfoque cuantitativo una primera indagación para identificar tendencias. Luego de los cálculos estadísticos descriptivos y análisis a los datos, se procedió a darle profundidad a la comprensión de las razones del incumplimiento; para ello se recurrió a la investigación cualitativa.

\section{Enfoque cuantitativo}

Se realizó un estudio transversal, con una muestra seleccionada a discreción bajo muestreos intencionados no probabilísticos, buscando representación de distintos tipos de empresas en varias regiones de Colombia, logrando recoger datos en 120 municipios. Se cumplieron los siguientes criterios de inclusión: gestores de SST con estudios y experiencia en el área, y desempeño actual como líderes del SG-SST.

El universo fue considerado como el total de graduados en salud ocupacional entre los años 2002 y 2013 en Colombia, que según el Observatorio Laboral de Educación fue de 35.468 personas.

La muestra estuvo conformada por seiscientas setenta y cinco (675) personas que cumplían con las siguientes características: el $38 \%$ eran hombres (255n) y el 62\% mujeres (420n); de 120 municipios de 32 departamentos del país, entre los que se destacaron: Antioquia 44\% (297n); Cundinamarca 14\% (95n); Valle 8,4\% (57n); Santander y Atlántico, 4,5\% cada uno (30n); Tolima, Nariño y Risaralda, 3,5\% cada uno (24n); y otros, 14,1\% (94n).

El total de la muestra tenía estudios en salud ocupacional. El 34\% (223n) era tecnólogo, el 28,4\% (192n) profesional, el 14,7\% (99n) técnico y el 16,37\% (113n) especialista. Los 6,53\% (48n) restantes eran magíster o bachilleres con énfasis en salud ocupacional. Laboraban en distintos sectores económicos: Servicios 22\% (148n),
Construcción 20,5\% (139n), Metalmecánico 16,2\% (109n), Salud $8 \%$ (54n), Agropecuario 6\% (41n) y otros sectores 27,3\% (184n).

\section{Enfoque cualitativo}

La unidad de análisis correspondió a nueve profesionales de salud ocupacional que previamente habian diligenciado el cuestionario; con ellos se realizaron grupos focales y entrevistas presenciales -desde un enfoque hermenéutico-; se buscó la comprensión y entendimiento de los sentidos de los discursos de los sujetos. En palabras de Echeverría ${ }^{18}$ el entendimiento a partir del diálogo y el ejercicio interpretativo.

Para asegurar el rigor y la credibilidad del estudio se cumplieron varios de los items contenidos en la lista de comprobación para la evaluación crítica de investigaciones cualitativas (COREQ) ${ }^{19}$, que aunque fue adoptada por revistas biomédicas presenta buenas prácticas que vale la pena copiar. Muy especialmente se siguieron las recomendaciones del análisis y procesamiento de los datos, en el sentido de: hacer explícito las categorías preconcebidas con que se realizó la codificación de los datos, obtener retroalimentación de los participantes sobre los resultados de la investigación antes de la publicación de este artículo y emplear software para el almacenamiento, la búsqueda y la codificación y análisis de los datos.

\section{Consideraciones éticas}

Este estudio cumplió con lo estipulado en la Resolución 8430 de 1993 de Colombia, la cual define este tipo de investigaciones como "sin riesgo". Está acorde además con los planteamientos de Emanuel $^{20}$ que, aunque se refieren a estudios clínicos, presenta recomendaciones que, en opinión del autor, todas las investigaciones deberían cumplir. De ahí el énfasis en el desarrollo de un estudio con valor social y validez científica, conveniente y transparente con la muestra.

\section{Instrumentos}

Para aumentar la fiabilidad y confianza de la interpretación de los hallazgos, se utilizó la triangulación metodológica, consistente en usar distintos instrumentos que confirmaran los resultados; es así como se emplearon: 675 cuestionarios con opción de múltiple respuesta -autoaplicables- a través de un Formulario de Google, tres grupos focales de 75 minutos y nueve entrevistas presenciales que duraron entre 50 y 70 minutos, grabadas y luego transcritas fielmente.

Para garantizar la consistencia y fiabilidad del estudio, los instrumentos fueron construidos a partir de la comprensión teórica de las categorías y sometidos a juicio de expertos -revisaron que el contenido de las preguntas diera cuenta de las categorias estudiadas y que el lenguaje y estilo de redacción fuera comprensibley prueba piloto.

Los instrumentos indagaron por estas dos preguntas: 1. ¿En su gestión como líder de SST, durante el último año, se ha sentido frustrado por no poder implementar los controles de seguridad que sabe que se requieren?; y, 2. ¿Cuáles han sido las razones para no implementar los controles de seguridad que sabe que se requieren? Para el caso del cuestionario se dieron opciones de respuesta obtenidas de referencia teórica- la muestra podía seleccionar varias o crear otras. (Ver ilustración 1).

\section{Análisis de datos}

Los datos cualitativos fueron fielmente registrados y luego transcritos. Con autorización de los participantes las sesiones de entre- 
Ilustración 1.

Cuestionario utilizado para la identificación de tendencias.

¿En su gestión como líder de SST, durante el último año se ha
sentido frustrado por no poder implementar los controles de
seguridad que sabe que se requieren?
Frustración entendida como un sentimiento de tristeza, decepción
y desilusión provocado por la imposibilidad de satisfacer una
necesidad o un deseo.
o Siempre
o Casi siempre
o A veces
o Casi nunca
o Nunca

¿Qué ha provocado que no pueda implementar los controles de seguridad que sabe deben hacerse?

Califique de 1 a 5 . Siendo 5 estar completamente de acuerdo con la afirmación.

\begin{tabular}{l|c|c|c|c|c|}
\hline $\begin{array}{l}\text { La falta de apoyo de la gerencia } \\
\text { 0 su jefe inmediato }\end{array}$ & 0 & 0 & 0 & 0 & 0 \\
$\begin{array}{l}\text { La falta de recursos económicos } \\
\text { de la empresa }\end{array}$ & 0 & 0 & 0 & 0 & 0 \\
$\begin{array}{l}\text { La falta de interés de la empresa } \\
\text { por proteger la seguridad de }\end{array}$ & 0 & 0 & 0 & 0 & 0 \\
\hline $\begin{array}{l}\text { los empleados } \\
\text { La falta de poder de decisión } \\
\text { de su cargo }\end{array}$ & 0 & 0 & 0 & 0 & 0 \\
$\begin{array}{l}\text { La imposibilidad de parar la } \\
\text { producción para implementar }\end{array}$ & 0 & 0 & 0 & 0 & 0 \\
\hline $\begin{array}{l}\text { los controles } \\
\text { La falta de apoyo del resto } \\
\text { de coordinadores de } \\
\text { procesos }\end{array}$ & 0 & 0 & 0 & 0 & 0 \\
\hline
\end{tabular}

\section{Otras razones}

vistas y grupos focales fueron grabadas. Más tarde procesadas con el software Atlas Ti. Se utilizó la técnica de análisis del discurso, identificación y clasificación estructural de dimensiones estratégicas y búsqueda de patrón de comportamiento común.

Para el análisis de los cuestionarios se efectuaron cálculos estadísticos descriptivos. La información se procesó con Microsoft Excel 2013. Se construyó una sábana de datos donde se vaciaron las respuestas de todos los ítems, logrando análisis por opción de respuesta y luego en forma conjunta.

\section{Limitaciones}

Este estudio solo consultó la opinión de los Gestores de Seguridad y Salud en el Trabajo (G-SST).

\section{RESULTADOS}

En el último año, el 23,11\% (156n) de los G-SST consultados casi siempre estuvo frustrado por no poder implementar los controles de seguridad laboral que sabía requería la empresa donde labora. Los motivos se resumen en seis razones, entre las que se destacan con un 31\% la falta de apoyo de la gerencia y un 27,1\% falta de apoyo del resto de coordinadores.

\section{Frustración profesional}

Entendida como un sentimiento de tristeza, decepción y desilusión provocado por la imposibilidad de satisfacer una necesidad o un deseo. El 4\% (27n) del total de la muestra manifestó que durante el último año se sintió siempre frustrado y el 19,11\% (129n) casi siempre frustrado, una permanente imposibilidad de realizar las funciones para las cuales fue contratado. El 50,67\% (342n) indicó que a veces se ha sentido frustrado. Un 17\% (115n) dijo que casi nunca y un 9,19\% (62n) durante el último año nunca se sintió frustrado.

Los niveles de frustración son similares entre G-SST hombres y mujeres. Los primeros registraron un 21\% en casi siempre sentirse frustrados, mientras que las mujeres un cercano 24\%. Al analizar los datos por regiones del país, se encuentra que la muestra del Valle del Cauca manifestó menor frustración. Por el contrario, Nariño registró los niveles más altos (45,8\%). (Ver Tabla 1).

No se observaron diferencias significativas por nivel académico. Los profesionales y especialistas presentaron un 24,7\% (89n) en casi siempre sentirse frustrados y los técnicos y tecnólogos un 21,7\% (78n).

\section{Razones para el incumplimiento}

Las encontradas corresponden al resorte y decisión de la empresa. El $58,1 \%$ se refiere específicamente al compromiso político de algún nivel de la empresa (alta dirección o coordinadores de procesos). Ver Tabla 2.

\section{DISCUSIÓN}

Esta investigación pretendió conocer las razones del incumplimiento de los controles de seguridad en el trabajo en empresas colombianas y encontró que en un 58,1\% se debe a falta de apoyo de la alta dirección y coordinadores de procesos. Estos resultados ratifican que el nivel de desarrollo en la gestión de los riesgos laborales es directamente proporcional a las condiciones internas que produzcan las empresas. Aunque haya legislación, organismos de control y asesores externos como las Aseguradoras de Riesgos Laborales si la empresa no se compromete no se lograrán resultados efectivos.

Los resultados son similares a otros estudios que develan que aún es necesario avanzar en la cultura de la seguridad laboral ${ }^{10}$ trascendiendo la documentación de los procesos al compromiso de la alta dirección, tal y como lo plantea el modelo de SG-SST propuesto por la Organización Internacional del Trabajo. ${ }^{2}$

Comparte la propuesta de que el compromiso debe ser de todas las partes interesadas. ${ }^{15,16}$ Se requiere un nuevo perfil de G-SST que no solo sea competente técnicamente en la identificación e intervención de los riesgos sino que también identifique la ubicación del área en el punto más alto de la empresa, es decir, una gestión estratégica $^{14}$, ya que según los resultados parecería ser que los profesionales no han sido convincentes y efectivos en hacer de la gestión de los riesgos una tarea de todos los miembros de la empresa.

Los resultados generan inquietud, porque a diferencia de otras profesiones, el sentir frustración por no hacer lo que corresponde puede ser la diferencia entre la vida y seguridad de otros. En Salud 
Tabla 1.

Resultados de frustración laboral de los gestores de seguridad y salud en el trabajo de las principales regiones de la muestra durante el último año.

\begin{tabular}{|c|c|c|c|c|c|c|c|c|c|c|c|c|c|c|}
\hline \multirow[t]{2}{*}{ Opciones de respuesta } & \multicolumn{2}{|c|}{ Antioquia } & \multicolumn{2}{|c|}{ Cundinamarca } & \multicolumn{2}{|c|}{ Valle del Cauca } & \multicolumn{2}{|c|}{ Atlántico } & \multicolumn{2}{|c|}{ Santander } & \multicolumn{2}{|c|}{ Tolima } & \multicolumn{2}{|c|}{ Nariño } \\
\hline & $\mathbf{n}$ & $\%$ & n & $\%$ & $\mathbf{n}$ & $\%$ & n & $\%$ & $\mathrm{n}$ & $\%$ & $n$ & $\%$ & $n$ & $\%$ \\
\hline$A$ veces & 155 & $52,2 \%$ & 55 & $57,9 \%$ & 31 & $54,4 \%$ & 15 & $50,0 \%$ & 17 & $56,7 \%$ & 12 & $50,0 \%$ & 9 & $37,5 \%$ \\
\hline Casi nunca & 48 & $16,2 \%$ & 11 & $11,6 \%$ & 17 & $29,8 \%$ & 5 & $16,7 \%$ & 3 & $10,0 \%$ & 3 & $12,5 \%$ & 1 & $4,2 \%$ \\
\hline Casi siempre & 55 & $18,5 \%$ & 16 & $16,8 \%$ & 7 & $12,3 \%$ & 5 & $16,7 \%$ & 6 & $20,0 \%$ & 4 & $16,7 \%$ & 7 & $29,2 \%$ \\
\hline Nunca & 28 & $9,4 \%$ & 11 & $11,6 \%$ & 1 & $1,8 \%$ & 1 & $3,3 \%$ & 4 & $13,3 \%$ & 3 & $12,5 \%$ & 3 & $12,5 \%$ \\
\hline Siempre & 11 & $3,7 \%$ & 2 & $2,1 \%$ & 1 & $1,8 \%$ & 4 & $13,3 \%$ & & $0,0 \%$ & 2 & $8,3 \%$ & 4 & $16,7 \%$ \\
\hline Total general & 297 & $100 \%$ & 95 & $100 \%$ & 57 & $100 \%$ & 30 & $100 \%$ & 30 & $100 \%$ & 24 & $100 \%$ & 24 & $100 \%$ \\
\hline$\%$ Nunca y casi nunca & 76 & $25,6 \%$ & 22 & $23,2 \%$ & 18 & $31,6 \%{ }^{*}$ & 6 & $20,0 \%$ & 7 & $23,3 \%$ & 6 & $25,0 \%$ & 4 & $16,7 \%$ \\
\hline \% Siempre y casi siempre & 66 & $22,2 \%$ & 18 & $18,9 \%$ & 8 & $14,0 \%$ & 9 & $30,0 \% *$ & 6 & $20,0 \%$ & 6 & $25,0 \%$ & 11 & $45,8 \% \%^{*}$ \\
\hline
\end{tabular}

* Resultado relevante. Fuente: Elaboración propia a partir de resultados.

Tabla 2.

Razones de no poder implementar los controles de seguridad laboral que se requieren por parte de las empresas en Colombia, según los gestores de seguridad y salud en el trabajo consultados.

\begin{tabular}{|c|c|c|c|c|c|c|c|c|c|c|}
\hline Opciones de respuesta & 1 & $n$ & 2 & $n$ & 3 & $n$ & 4 & $n$ & $5^{*}$ & $n$ \\
\hline Falta de apoyo de la gerencia & $12,1 \%$ & 82 & $8,9 \%$ & 60 & $20,9 \%$ & 141 & $27,1 \%$ & 183 & $31,0 \%$ & 209 \\
\hline Falta de recursos económicos & $12,7 \%$ & 86 & $12,6 \%$ & 85 & $25,2 \%$ & 170 & $26,2 \%$ & 177 & $23,3 \%$ & 157 \\
\hline Falta de interés de la empresa & $16,9 \%$ & 114 & $14,2 \%$ & 96 & $19,4 \%$ & 131 & $28,7 \%$ & 194 & $20,7 \%$ & 140 \\
\hline Falta de poder de decisión del cargo & $23,7 \%$ & 160 & $16,1 \%$ & 109 & $17,6 \%$ & 119 & $22,4 \%$ & 151 & $20,1 \%$ & 136 \\
\hline Imposibilidad de parar la producción & $16,0 \%$ & 108 & $13,0 \%$ & 88 & $24,9 \%$ & 168 & $22,7 \%$ & 153 & $23,4 \%$ & 158 \\
\hline Falta de apoyo del resto de coordinadores & $13,5 \%$ & 91 & $10,7 \%$ & 72 & $22,8 \%$ & 154 & $25,9 \%$ & 175 & $27,1 \%$ & 183 \\
\hline Total frecuencias & & 641 & & 510 & & 883 & & 1033 & & 983 \\
\hline$\%$ del total de frecuencia & & $15,8 \%$ & & $12,6 \%$ & & $21,8 \%$ & & $25,5 \%$ & & $24,3 \%$ \\
\hline
\end{tabular}

*Califique de 1 a 5 . Siendo 5 estar completamente de acuerdo con la afirmación. Fuente: Elaboración propia a partir de resultados

Ocupacional (SO), esos niveles de frustración se traducen en desmejoras de la calidad de vida laboral y bienestar de cientos de empleados del país.

Este estudio genera importantes contribuciones. En el sentido académico abre nuevos interrogantes que se recomienda indagar. Por ejemplo: ¿cuál es la visión de los empresarios frente a las razones de la no implementación de los controles?; ¿qué tanto la falta de apoyo de la alta dirección y los equipos de trabajo es una demostración de la baja competencia estratégica de los G-SST consultados?; ¿por qué tan poco apoyo de la alta dirección y coordinadores de proceso?; y, los resultados del Valle del Cauca, ¿están relacionados con ser la región del país con más estudios científicos en el área? ${ }^{21}$

A nivel práctico llama la atención de las empresas por cuanto que cumplir con la SO supera la contratación de un profesional para que lidere el proceso: se trata de acompañarlo en la generación de cultura de seguridad a todos los niveles de la compañía. Abre la discusión frente a por qué los G-SST no han logrado posicionarse como un actor estratégico dentro de la organización e incidir en la toma de decisiones, tal vez una asignatura pendiente de los líderes del área que deben superar lo asistencial de sus interven- ciones, pensar estratégicamente su desempeño y lograr la confianza y apoyo de la alta gerencia.

\section{CONCLUSIONES}

A partir de esta investigación se concluye que el SG-SST requiere ser implementado con una visión más estratégica por parte de las empresas y una acción más convincente por parte de los profesionales del área. Las razones expuestas para no cumplir los controles de seguridad son todas del resorte de la organización y pueden ser superadas si se realiza un trabajo sinérgico entre todos los grupos interesados.

A nivel teórico se ha planteado una estrecha relación entre el éxito en la gestión de los riesgos y el nivel de compromiso de la alta dirección y de allí el aporte de este estudio, pues al parecer la principal mejora que tendría que hacerse es el incremento en este aspecto. A nivel metodológico se logró resolver el objetivo, los instrumentos dieron cuenta de las dimensiones abordadas. Se sugiere en nuevos estudios ampliar la muestra a las Aseguradoras de Riesgos Laborales y empresarios. 


\section{REFERENCIAS}

1. Organización Mundial de la Salud-OMS. Estrategia mundial de la salud ocupacional para todos. El camino hacia la salud en el trabajo. Helsinki: OMS; 1995.

2. Organización Internacional del Trabajo-OIT. Directrices relativas a los sistemas de gestión de la seguridad y la salud en el trabajo. Ginebra: 0IT; 2001.

3. Creus SA, Mangusio J. Seguridad e higiene en el trabajo: un enfoque integral. Buenos Aires: Alfaomega; 2011.

4. Pater R. Three Essentials for Elevating Safety Culture. Occup Health Saf. 2015; 84:6-106.

5. Organización Internacional del Trabajo-OIT. Investigación de accidentes del trabajo y enfermedades profesionales. Ginebra: OIT; 2015.

6. Asociación Nacional de Empresarios de Colombia-ANDI. Mercado Laboral Colombiano [en línea]. Bogotá: ANDI; 2012 [consultado 6 jun 2014]. Disponible en: http://www.andi.com.co/Archivos/file/Asamblea/2012/02Jueves_PM/ RafaelPardo_MTrabajo.pdf

7. Colombia. Ministerio de Trabajo. Disminuyen muertes por accidentalidad laboral [en linea]. Bogotá: Ministerio de Trabajo; 2015 [consultado abr 2016]. Disponible en: http://www.mintrabajo.gov.co/abril-2015/4398-disminuyenmuertes-por-accidentalidad-laboral.html

8. Lizarazo C, Fajardo J, Berrio S, Quintana L. Breve historia de la salud ocupacional en Colombia. Arch prev riesgos Ibor. 2011; 14(1):38-42.

9. Torres-Rey C, Conde-Sierra J, Checa-Guerrero D, Díaz-Criollo S, Palma-Parra R, Varona-Uribe M. Servicios de medicina del trabajo en Colombia. Rev Salud Pública (Bogotá). 2012; 14(4):598-606.

10. Ministerio del Trabajo. Informe ejecutivo II encuesta nacional de condiciones de seguridad y salud en el trabajo en el sistema general de riesgos. Bogotá: Grafiq Editores S.A.S.; 2013.

11. Galloway S. Business and safety: are the strategies aligned? Occup Health Saf. 2014; 83(11):62.

12. Fernández-Muñiz B, Montes-Peón J, Vázquez-Ordás C. Safety leadership, risk management and safety performance in Spanish firms. Safety Sci. 2014; (70):295-307.
13. Vega-Monsalve NC. Competencias laborales y gestión de riesgos ocupacionales. Congreso Internacional Salud \& Seguridad en el Trabajo; 22-23 may 2014; Medellin, Colombia [en línea]. Medellin: Uniminuto-Seccional Bello; 2014 [citado jun 2016]. Disponible en: http://www.uniminuto.edu/documents/ 991974/2604818/Ninfa+Vega+OK+Version+para+publicar.pdf/ce1c257ef720-409c-bd7d-1ae6e38a6e71.

14. Vega-Monsalve NC. Neocompetencias. Nuevo enfoque de competencias laborales en Salud Ocupacional. Educac Méd Super [en línea]. 2016 [citado jun 2016]; 30(3):00. Disponible en: http://www.ems.sld.cu/index.php/ems/ article/view/802/428

15. Fernández-Muñiz B, Montes-Peón J, Vásquez-Ordás C. Relation between occupational safety management and firm performance. Safety Sci. 2009 ; (47):980-991.

16. Chen JK, Zorigt D. Managing occupational health and safety in the mining industry. J Bus Res. 2013; 66(11):2321-2331.

17. Saracino A, Curcuruto M, Antonioni G, Mariani M, Guglielmi D, Spadoni G. Proactivity-and-consequence-based safety incentive (PCBSI) developed with a fuzzy approach to reduce occupational accidents. Safety Sci. 2015; (79):175-183.

18. Echeverria R. El Búho de Minerva. Santiago: Dolmen; 1997.

19. Tong A, Sainsbury $P$, Craig J. Consolidated criteria for reporting qualitative research (COREQ): A 32-item checklist for interviews and focus groups. Inter J Qual Health Care. 2007; 19(6):349-357.

20. Emanuel E. ¿Qué Hace Que la Investigación Clínica Sea Ética? Siete Requisitos Básicos. En: Pellegrini Filho A, Macklin R. Investigación en Sujetos Humanos: experiencia Internacional. Santiago: Organización Mundial de la Salud-OMS; 1999. pp. 83-95. (Cuadernos del Programa Regional de Bioética).

21. Beltrán CP. Aportes de postgrados seleccionados a los diferentes ámbitos de salud ocupacional en Colombia [tesis]. Bogotá: Pontificia Universidad Javeriana; 2012. 